\title{
EXPERIMENTAL STUDY ON THE 5TH ORDER NONLINEAR RESONANCE AT TAIWAN LIGHT SOURCE
}

\author{
T. S. Ueng, Jenny Chen, K. T. Hsu, K. H. Hu, C. C. Kuo, SRRC, Hsinchu, Taiwan
}

\begin{abstract}
The 5th order nonlinear resonance was studied at SRRC's electron storage ring, which has working tunes around $v_{x}=7.23$ and $v_{y}=4.17$. This resonance is interesting due to the possible systematic errors from its 6-fold symmetry lattice design. In order to understand the dynamics of resonance and effects of betatron decoherence and linear coupling, experiments have been performed using the 2-BPM turn-by-turn method and phase space maps to study its phenomena. The experimental results are presented.
\end{abstract}

\section{INTROCUCTION}

Nonlinear beam dynamics experiments have been performed at many accelerator facilities for various resonance phenomena at different resonance conditions $[1,2,3,4]$. The sextupoles, octupoles and higher order multipoles in the accelerator can produce the non-linear effects that perturb the beam orbit and give the phase space significantly different from that with linear lattice elements. When the betatron tune is near a resonance condition, $\boldsymbol{m} v_{\mathrm{x}}+\boldsymbol{n} \boldsymbol{v}_{\mathrm{y}}=\boldsymbol{\ell}$, where $\boldsymbol{m}, \boldsymbol{n}, \boldsymbol{\ell}$ are integers, its Poincaré map shows deviation from a simple ellipse.

The Taiwan Light Source (TLS) electron storage ring of SRRC is a 3rd generation synchrotron radiation source providing low emittance electron beam. It requires strong transverse focusing and defocusing quadrupoles and strong chromaticity correcting sextupoles to achieve a good performance. A series of studies on the nonlinear resonance[5] have been conducted recently to understand its effects. The TLS is a storage ring having a superperiodicity of 6 . It operates routinely with transverse betatron tunes at around $v_{x}=7.23$ and $v_{y}=4.17$. At the one dimensional nonlinear resonance, $\mathrm{mv}_{\mathrm{x}}=\boldsymbol{\ell}$. The 5 th order resonance with $\mathrm{m}=5$ and $v_{\mathrm{x}}=7.2$ gives $\boldsymbol{\ell}=36$. It is just a multiple of the superperiodicity. In this experiment, the effects of sextupoles and the decapole in the dipole magnet on the 5 th order systematic resonance is studied.

\section{EXPERIMENTAL ARRANGEMENT}

The data acquisition started by kicking the electron beam horizontally with one of the injection kickers, which have also been configured for the turn-by-turn experiments. Various kicking strengths have been applied from minimum and increased until some of the electrons would be kicked out of the storage ring. The centroid of the kicked beam has been measured by two beam position monitors which separated about 90 degrees of phase advance and similar betratron amplitude functions. The signals from the beam position monitors were processed with Bergoz's Log-Ratio beam position monitor electronics (LR-BPM). Its output was fed into the VME based transient digitizers and, then, the workstation for processing. A GUI based software was developed for the online data acquisition. The final turn-by-turn position data was also saved for detailed off-line analysis. Even the data acquisition could be done with a single bunch beam, during this series of experiments the multi-bunch beam was used to avoid a time consuming single bunch injection process. Also, with the higher beam current it seemed to give a better resolution in the beam position.

In the process to obtain the 5th order resonance data, several conditions must be met and some parameters were necessary for the data acquisition and analysis. During the experiments we have also made detailed studies on these conditions and parameters, such as decoherence, chromaticity, tune variation with betatron amplitude, and coupling effects. Its results are also described in the following paragraphs.

\section{RESULT AND DISCUSSION}

\subsection{Decoherence}

Due to the spread of the betatron tune within the electron beam, the transversely kicked beam will decohere as the individual betatron phase of electrons disperse. Since the beam position monitors detected only the centroid of the beam, the turn-by-turn beam positions will show a decaying oscillation. Its oscillation amplitude could decay very rapidly as the kicked amplitude was increased. There are two major sources contributed to the tune spread. One is from the energy spread of the beam coupled to the betatron tune through the chromaticity. Another is from the transverse nonlinearity. During the experiments, the data were taken by adjusting the sextupoles in order to have a close zero chromaticity to reduce the effect of decoherence. But a strong decoherence from the nonlinearity was still shown on our data. It can be seen in Fig. 1 that the envelope of the turnby-turn beam positions decreased quickly as the kicking strength increased.

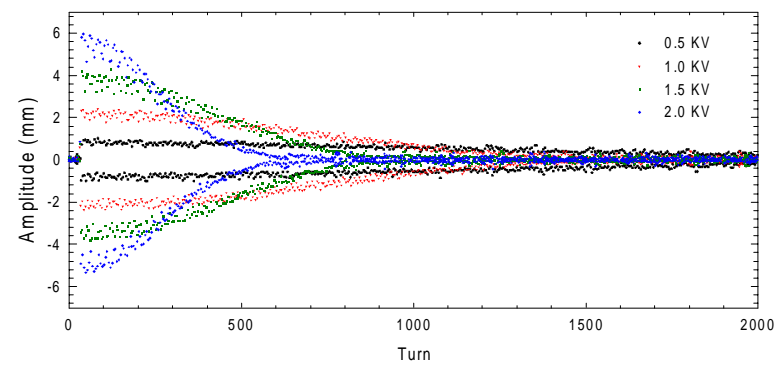

Figure 1: The envelopes of the turn-by-turn beam positions show the beam positions damped quickly as the kicking strength increased even when the chromaticity is set to about zero. 


\subsection{Chromaticity}

There are two circuits of sextupoles used to adjust the chromaticities, one for focusing and another for defocusing. The chromaticities were obtained first by measuring betatron tune $(v)$ vs. the RF frequency $\left(\omega_{\mathrm{rf}}\right)$ at selected sextupole strengths. Then, the chromaticity was obtained with the relation $\xi=-\eta \mathrm{d} v /\left(\mathrm{d} \omega_{\mathrm{rr}} / \omega_{\mathrm{rf}}\right)$, where $\eta$ is the phase-slip factor. Before each 5th order resonance measurement, we would set the proper sextupole currents to have chromaticities about zero to take the data.

\subsection{Amplitude Dependent Tune Shift}

The amplitude dependent tune shift was measured with turn-by-turn method at $v_{x} \sim 7.21$ for several selected chromaticities. At each chromaticity, the beam positions were also recorded for various kicking strengths. The fast fourier transform (FFT) spectrum of beam positions for each kicking strength was used to obtain the peak corresponding to the tune. This peak was fitted with a Lorentzen distribution function to find the center of the peak and its full width at half maximum(FWHM). Each set of turn-by-turn beam positions at the two beam position monitors were used to construct a phase space ellipse and fit to the following equation to obtain $\left(\beta_{1} / \beta_{2}\right)^{1 / 2}$, $\psi_{12}$ and $\mathrm{J}$, where $\beta_{1}$ and $\beta_{2}$ are the betatron amplitude functions at the two BPM positions, $\psi_{12}$ is the phase advance between these 2 BPMs and $J$ is the conjugate action variable.

$$
\mathrm{x}_{1}^{2}+\left(\sqrt{\frac{\beta_{1}}{\beta_{2}}} \cdot \csc \psi_{21} \cdot \mathrm{x}_{2}-\cot \psi_{21} \cdot \mathrm{x}_{1}\right)^{2}=2 \beta_{1} \mathrm{~J}
$$

The obtained results were: $\left(\beta_{1} / \beta_{2}\right)^{1 / 2}=1.02 \pm 0.01$, $\psi_{12}=86.1^{\circ} \pm 0.2^{\circ}$. These fitted parameters were also compared with those obtained at $v_{x} \sim 0.2$ at smaller kicking strength. The results were consistent within uncertainty. The relation between the tune and $\mathrm{J}$ at different chromaticity is shown in the top of Fig. 2. The relation between the FWHM of the tune peak and $\mathrm{J}$ is also shown in the bottom of Fig. 2. The slope shown in the top plot of Fig. 2 for the different chromaticities of 1.5, 0.8, $0.4,0.3$ and -0.7 are $-1.26 \times 10^{-3},-1.15 \times 10^{-3},-1.11 \times 10^{-3}$, $-1.03 \times 10^{-3}$ and $-0.74 \times 10^{-3}\left(\mathrm{~mm} \mathrm{mrad}^{-1}\right.$, respectively. The major source of uncertainty was from the measurement of chromaticity which was about \pm 0.15 . These slopes could be used as the nonlinear detuning parameters, $\alpha_{x x}$. During the measurement, all of the parameters were fixed except the chromaticity changed. Fig. 2 also shows that the tune of the storage ring was affected by the changing the sextupole strength.

\subsection{Coupling}

During the experiment the kicker used was one of the injection kickers. It was designed to kick only in the horizontal plane. Due to the alignment errors in the magnet elements in lattice, there exists some coupling between the horizontal and the vertical motion of the electron beam. There were 2 skew quadrupoles installed in the storage ring to control the coupling between these two planes. During the experiments, the strengths of the quadrupoles were adjusted to suppress the coupling. From the FFT spectrum of the turn-by-turn horizontal beam positions acquired during the experiments, the effect of coupling was estimated generally less than $1 \%$.

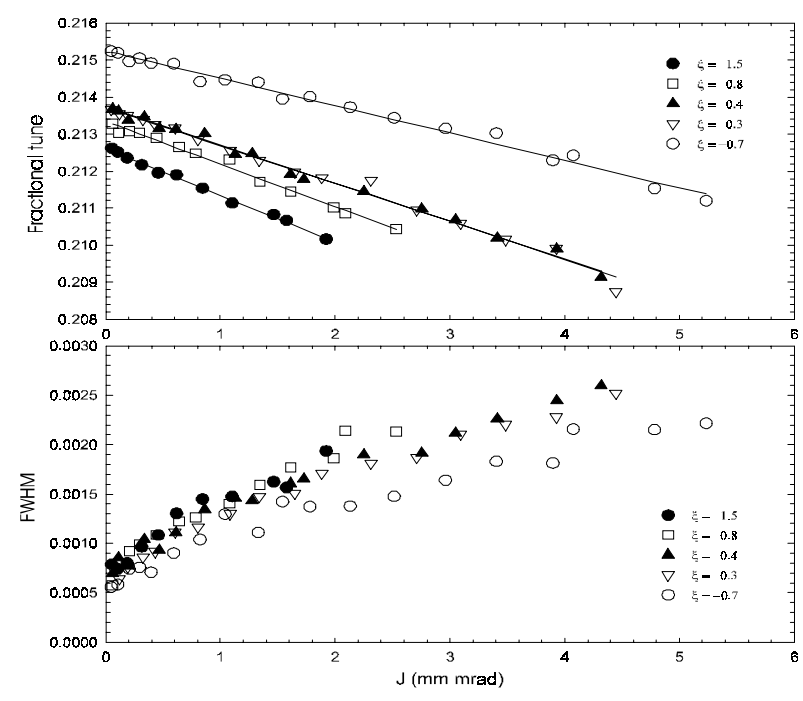

Figure 2: (Top) The relation between the center of the tune peak and the action variable J. (Bottom) The relation between the FWHM of the tune peak and J.

\subsection{5th Order Resonance}

Before taking the 5th order resonance data, the chromaticities were adjusted to close to zero and the coupling between the horizontal and the vertical motion was also suppressed to minimum. The horizontal tune was set to be slightly above $v_{x}=7.2$ with the GUI based online data acquisition software. Then, the electron beam was kicked with the injection kicker starting from small kicking strength, and increased gradually. The tune would be decreased due to the effect of tune decreasing with increasing amplitude. The effect of decoherence was increased with increased amplitude, too. The horizontal tune of electrons would be exactly 7.2 at some amplitude. Five oval curves or spots could be seen in the raw phase space plot. It showed that the electrons were locked on the tune of exactly 7.2, thus, 5 islands appeared. In Fig. 3, the 32000 turn-by-turn beam positions (about $13 \mathrm{~ms}$ ) at one beam position monitor are shown. It shows that the electrons could overcome the decoherence and the radiation damping (about $10 \mathrm{~ms}$ at TLS) and stay at these islands for a long period of time. Right after the kicking the electrons seemed to move around stable fixed points with slightly larger amplitude. Another slowly decreasing of the beam positions toward the center of the electron beam shown in Fig. 3 could be due to the effect of the radiation damping and the residual effect of the decoherence. As the kicking strength increased again, the islands disappeared. A strong decoherence effect showed up again and a pentagon shape shown in the phase space plot with a quick damp of electron beam positions into the orbit center. The FFT spectrum of the beam positions can give the fractional tune. Applying the beam position 
data, when electrons were kicked into islands, with FFT, the side band of the peak corresponding to $v_{x}=7.2$ will give the island tune. From our massive data, the islands tune shows a dependence on the resonance proximity parameter, $\delta_{x}$. It also implies the island tune depends on the kicking amplitude. At present the magnet power supply ripple at SRRC is about $100 \mathrm{ppm}$. It will not affect the present routine operation. But, the minor change in tune by this small ripple seemed to be able to affect the resonance proximity parameter during the nonlinear experiment. It would make the interpretation of data difficult. A detailed study is under way to investigate the dynamics of the resonance islands.

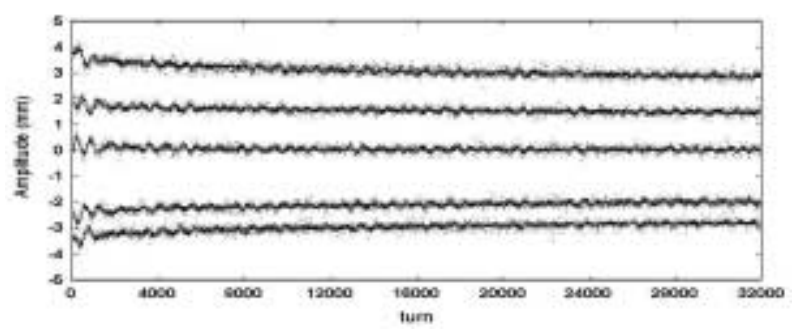

Figure 3: 32000 turn beam positions vs. turn number

The Hamiltonian near the one-dimensional 5th order resonance can be expressed as

$$
\tilde{H}=\delta \cdot J_{x}+\frac{1}{2} \alpha_{x x} \cdot J_{x}^{2}+G_{5,0, l} J_{x}^{5 / 2} \cos 5 \phi
$$

where $\delta=v_{\mathrm{x}}-\ell / 5$ is the resonance proximity parameter, $\phi$ $=\phi_{\mathrm{x}}-\ell / 5 \cdot \theta+\xi / 5, \mathrm{~J}_{\mathrm{x}}$ and $\phi_{\mathrm{x}}$ are conjugate action-angle variables. $G$ and $\xi$ are the resonance strength and phase, $\theta$ is the orbital angle. One set of the raw phase space plot is shown in Fig. 4. In the plot only the first 200 turns beam positions after kicking are drawn for the small amplitude ellipses and first 120 turns for those above the resonance islands. But, the two island runs each contains 2000 turns. It shows clearly the evolution of the phase space ellipse as the kicking amplitude increases. Its corresponding map in action-angle variables is shown in Fig. 5.

\section{CONCLUSION}

The 5th order resonance at $v_{\mathrm{x}}=7.2$ was studied experimentally recently in SRRC. Later, we will investigate the dynamics of resonance islands and compare the result with the computer modeling. The 5th order Hamiltonian will also be investigated in order to understand this resonance analytically. Also, during the present experiment, the multi-bunch beam was used. Many complicated beam dynamics mechanism might show up in the acquired data, which makes the explanation of the data difficult. We are planning to run the experiment with the single bunch beam to study the resonance properties. The data acquisition hardware is also under improvement in order to study the vertical dimension resonance and the 2-dimensional resonance.

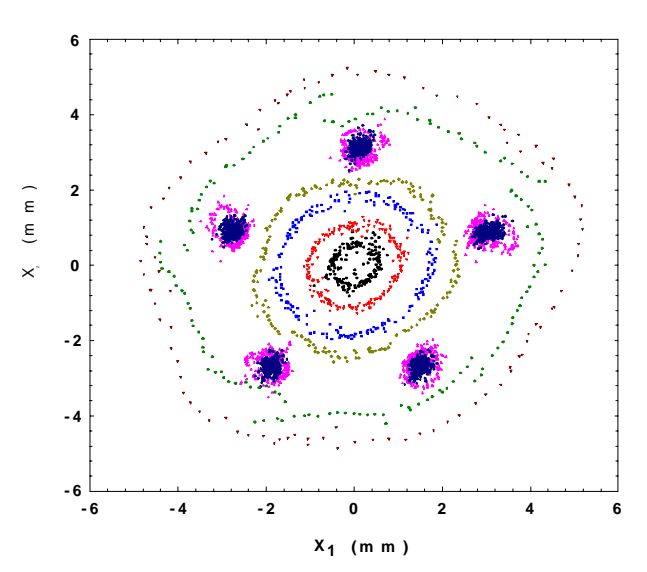

Figure 4: The phase space map at betatron tune $v_{x}=7.2$.

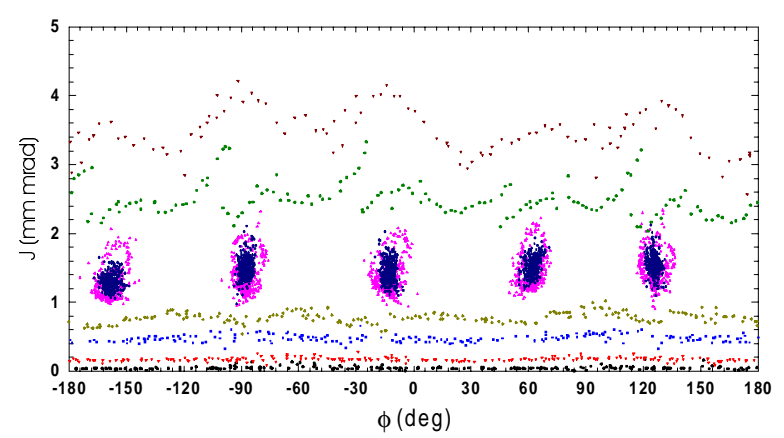

Figure 5: The Pioncaré map of data in Fig. 4 in actionangle variables.

\section{ACKNOWLEDGEMENT}

The authors would like to thank the members of the SRRC Operation Group for their assistant in the operation of the accelerator during the experiment. Our heartfelt appreciation should go to Prof. S. Y. Lee of IUCF. Without Prof. Lee's motivation and guidance this work would not be completed.

\section{REFERENCES}

[1] A. Chao et al., "Experimental Investigation of Nonlinear Dynamics in the Fermilab Tevatron," Phys. Rev. Lett.. 61, 2752, 1988.

[2] S.Y. Lee et al., "Experimental Determination of a Nonlinear Hamiltonian in a Synchrotron," Phys. Rev. Lett.. 67, 3768, 1991.

[3] S.Y. Lee, "Review on Nonlinear Beam Dynamics Expt.," Inst. Phys. Conf. Ser. No 131, 249, 1993.

[4] Y. Wang et al., "Effects of Tune Modulation on Particles Trapped in One-dimensional Resonance Islands," Phys. Rev. 49, 5697, 1994.

[5] C.C. Kuo et al., "Nonlinear Beam Dynamics Experiments at the SRRC," these proceedings. 